\title{
Fast rate of evolution in alternatively spliced coding regions of
} mammalian genes

\author{
Ekaterina O Ermakova*1,2, Ramil N Nurtdinov ${ }^{1}$ and Mikhail S Gelfand ${ }^{1,2}$
}

\author{
Address: ${ }^{1}$ Department of Bioengineering and Bioinformatics, Moscow State University, Vorob'evy gory, 1-73, 119992, Moscow, Russia and \\ ${ }^{2}$ Research and Training Center "Bioinformatics", Institute for Information Transmission Problems, Russian Academy of Sciences, Bolshoi Karetny \\ per. 19, 127994, Moscow, Russia \\ Email: Ekaterina O Ermakova* - ermakova@iitp.ru; Ramil N Nurtdinov - n_ramil@mail.ru; Mikhail S Gelfand - gelfand@iitp.ru \\ * Corresponding author
}

Published: 18 April 2006

BMC Genomics2006, 7:84 doi:10.1/86/147|-2164-7-84

This article is available from: http://www.biomedcentral.com/I47/-2/64/7/84

(c) 2006Ermakova et al; licensee BioMed Central Ltd.

This is an Open Access article distributed under the terms of the Creative Commons Attribution License (http://creativecommons.org/licenses/by/2.0), which permits unrestricted use, distribution, and reproduction in any medium, provided the original work is properly cited.
Received: 21 December 2005

Accepted: 18 April 2006

\begin{abstract}
Background: At least half of mammalian genes are alternatively spliced. Alternative isoforms are often genome-specific and it has been suggested that alternative splicing is one of the major mechanisms for generating protein diversity in the course of evolution. Another way of looking at alternative splicing is to consider sequence evolution of constitutive and alternative regions of protein-coding genes. Indeed, it turns out that constitutive and alternative regions evolve in different ways.

Results: A set of 3029 orthologous pairs of human and mouse alternatively spliced genes was considered. The rate of nonsynonymous substitutions $\left(d_{N}\right)$, the rate of synonymous substitutions $\left(d_{S}\right)$, and their ratio $\left(\omega=d_{N} / d_{S}\right)$ appear to be significantly higher in alternatively spliced coding regions compared to constitutive regions. When $\mathrm{N}$-terminal, internal and $\mathrm{C}$-terminal alternatives are analysed separately, C-terminal alternatives appear to make the main contribution to the observed difference. The effects become even more pronounced in a subset of fast evolving genes.

Conclusion: These results provide evidence of weaker purifying selection and/or stronger positive selection in alternative regions and thus one more confirmation of accelerated evolution in alternative regions. This study corroborates the theory that alternative splicing serves as a testing ground for molecular evolution.
\end{abstract}

\section{Background}

Alternative splicing is a major mechanism for generating functional and evolutionary diversity of proteins in mammals [1,2], for a review see [3]. Indeed, alternative splicing allows for generation of novel proteins without sacrificing old ones [2]. If a new isoform proves to be beneficial, its fraction increases by subtle regulatory changes. On the other hand, unlike gene duplication, alternative splicing does not lead to dramatic changes in protein concentrations. Moreover, it has been demonstrated that duplicated genes are rarely alternatively spliced compared to singletons $[4,5]$.

There are good reasons to believe that some key mutational events driving evolution might reside in introns, untranslated regions (UTRs) and/or nontranscribed regulatory regions [6-8]. A large fraction of alternative splicing events occur in untranslated regions [9]. Nevertheless, most studies of molecular evolution have focused on the analysis of protein coding regions, as these data are sim- 
pler to obtain and are more amenable to functional interpretation.

From this point of view, alternative regions of genes occupy an intermediate position. Alternatively spliced regions are often evolutionary young: indeed, about a half of genes in human-mouse orthologous pairs have speciesspecific isoforms $[2,10]$. In many respects, constitutive and alternative regions are organized in different ways. Alternative human splice sites are on the average weaker than constitutive ones [11]. Non-canonical GC-AG introns tend to be alternative [12]. Among human exons conserved in mouse, about $77 \%$ of alternative cassette exons are flanked on both sides by long conserved intronic sequences, compared to only $17 \%$ of the constitutive exons [13]. Overall, statistical and evolutionary features of constitutive and alternative exons are sufficiently different to provide for computational recognition of these exons [14-16].

In several early studies it has been observed that patterns of nucleotide substitutions are different in alternative and constitutive coding regions. Iida and Akashi [17] analysed 26 pairs of alternatively spliced human genes and their non-human mammalian orthologs and demonstrated that synonymous divergence was lower and the nonsynonymous divergence was higher in alternative regions compared to constitutive regions. Evidence for diversifying selection was observed in alternative regions of CD45 [18], whereas the reduced rate of synonymous substitutions in an alternative region of BRCA1 [19] was assigned to purifying selection due to exonic splicing enhancer sites [20]. Recently, lower synonymous divergence in alternative exons compared to constitutive ones was demonstrated in a large-scale study of human, chimpanzee, mouse and rat genes [21].

Here we analyze evolutionary patterns in a set of 3029 pairs of orthologous human and mouse genes. We consider all types of alternative splicing and analyze separately 5 '-, internal, and 3 '-regions of genes, as well as faster and slower evolving genes.

\section{Results}

We considered 3029 alternatively spliced human genes and their mouse orthlogs (Figure 1). The sample was divided into three bins of equal size with respect to nucleotide identity in coding regions. Nucleotide alignments of coding regions were sliced into constitutive (C) and alternative (A) fragments. Alternative fragments were further sorted into $\mathrm{N}$-terminal (AN), internal (AI), and C-terminal (AC). To reveal the general pattern of evolution in these regions, we estimated the amino acid identity (Id), the nonsynonymous substitution rate $\left(\mathrm{d}_{\mathrm{N}}\right)$, the synonymous substitution rate $\left(\mathrm{d}_{\mathrm{S}}\right)$, and $\omega=\mathrm{d}_{\mathrm{N}} / \mathrm{d}_{\mathrm{S}}$ (Table 1 ). Glo-

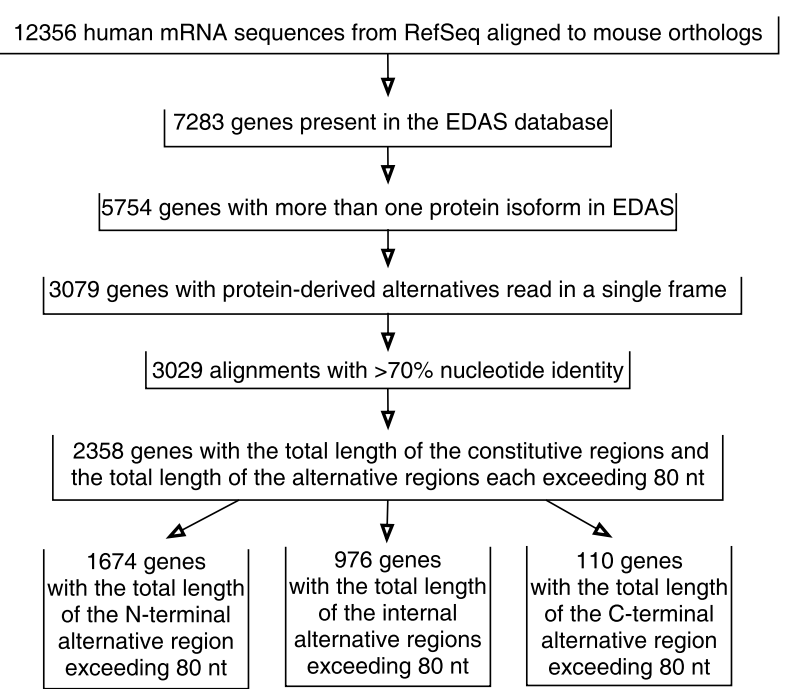

\section{Figure I}

The data flow through the analysis pipeline.

bal meta-alignments (concatenated alignment fragments across all considered genes and across three rate bins, see Methods) of five types (C, A, AN, AI, AC) were used. We performed 2000 bootstrap replications to evaluate the robustness of our estimates (Figures 2, 3, 4, 5).

It turned out that $\operatorname{Id}(\mathrm{A})<\operatorname{Id}(\mathrm{C}), \mathrm{dN}(\mathrm{A})>\mathrm{dN}(\mathrm{C})$, and $\omega(\mathrm{A})>\omega(\mathrm{C})$ for alternatively spliced genes irrespective of the rate of evolution. These results show that negative selection is weaker and/or positive selection is stronger in alternative regions and thus confirm that alternatively spliced coding regions are hotspots of molecular evolution. Unexpectedly, $\mathrm{dN}$ and $\omega$ rise dramatically at the Cterminal alternative regions (Figures 3 and 5).

The pattern of synonymous substitutions is more complicated, as it depends on the rate of evolution (Figure 4). The general pattern is that $\mathrm{d}_{\mathrm{S}}$ in alternative regions increases in the $5^{\prime}$ to $3^{\prime}$ direction. In genes evolving at the medium rate, $d_{S}(A N)<d_{S}(A I)<d_{S}(A C)$, whereas in fast evolving genes $d_{S}(A I)>d_{S}(A C)$.

For control, we considered N-terminal and C-terminal constitutively spliced regions and performed similar analysis. All computed evolutionary parameters were the same as in the constitutive regions in general (data not shown). Thus the observed difference cannot be explained simply by faster evolution at gene termini. 


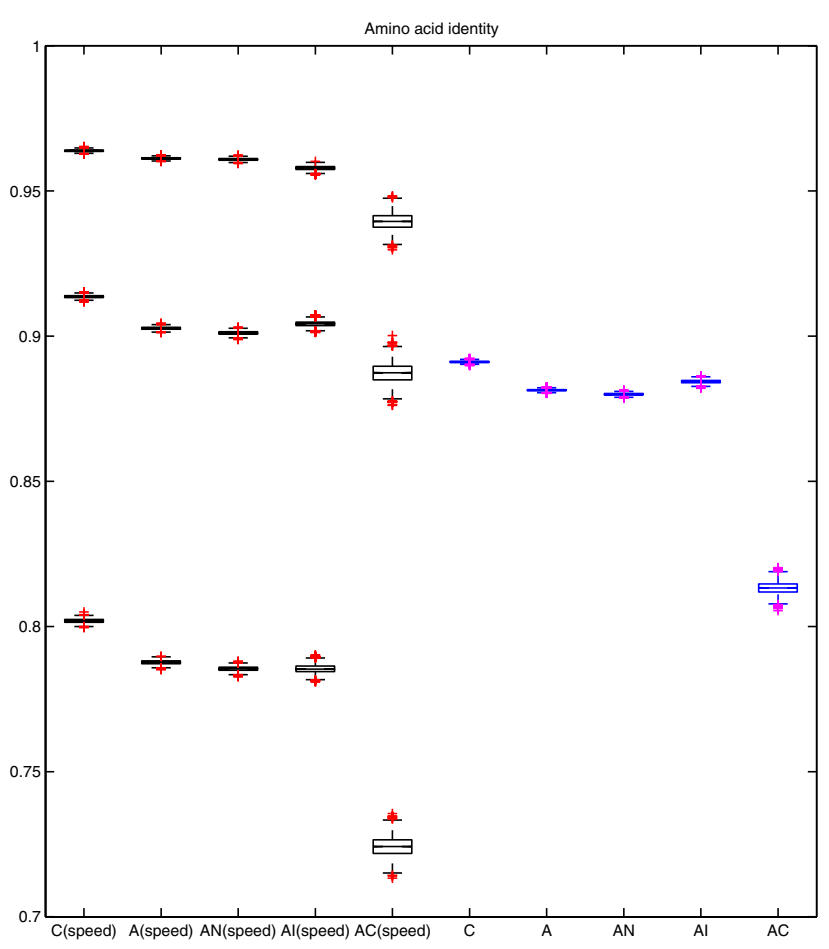

Figure 2

Amino acid identity in alignments of constitutive and alternative coding regions of $\mathbf{3 0 2 9}$ alternatively spliced genes classified by the overall evolution rate. $\mathrm{C}-$ constitutive regions. $\mathrm{A}-$ alternative regions. $\mathrm{AN}-\mathrm{N}$ terminal alternative regions. $\mathrm{Al}-$ internal alternative regions. AC - C-terminal alternative regions. Five columns on the left show results for three equal bins of slowly evolving genes, genes evolving with medium speed, and fast evolving genes. Five columns on the right correspond to the total sample of 3029 alternatively spliced genes. To estimate the average amino acid identity, we used global meta-alignments (see Methods). The boxplots represent the results for 2000 bootstrap replications.

Next, we analyzed individual gene pairs. For each of 2358 genes with the total lengths of constitutive and of alternative regions both exceeding $80 \mathrm{bp}, \omega$ for constitutive $\left(\omega_{\mathrm{C}}\right)$ and alternative $\left(\omega_{\mathrm{A}}\right)$ regions was calculated separately. Figure 6 a represents the distribution of the difference $\left(\omega_{\mathrm{C}^{-}}\right.$ $\left.\omega_{A}\right)$. The distribution is skewed, showing that $\omega$ tends to be greater in alternative regions. We used the chi-squared test to compare the distributions of $\left|\omega_{\mathrm{C}}-\omega_{\mathrm{A}}\right|$ in the case $\omega_{C}>\omega_{A}$ and in the case $\omega_{C}<\omega_{A}$. The null hypothesis that the distributions were the same was rejected at the significance level $10^{-7}$. When $\mathrm{N}$-terminal, internal, and C-terminal alternatives were considered separately, the effect was the same (Figure $6 \mathrm{~b}-\mathrm{d}$ ). The null hypothesis that the distribution of $\left|\omega_{\mathrm{C}}-\omega_{\mathrm{AN}}\right|$ was symmetrical was rejected at the significance level $10^{-9}$, the one that the distribution of $\mid \omega_{\mathrm{C}^{-}}$ $\omega_{\mathrm{AI}} \mid$ was symmetrical, at the significance level $10^{-3}$, and

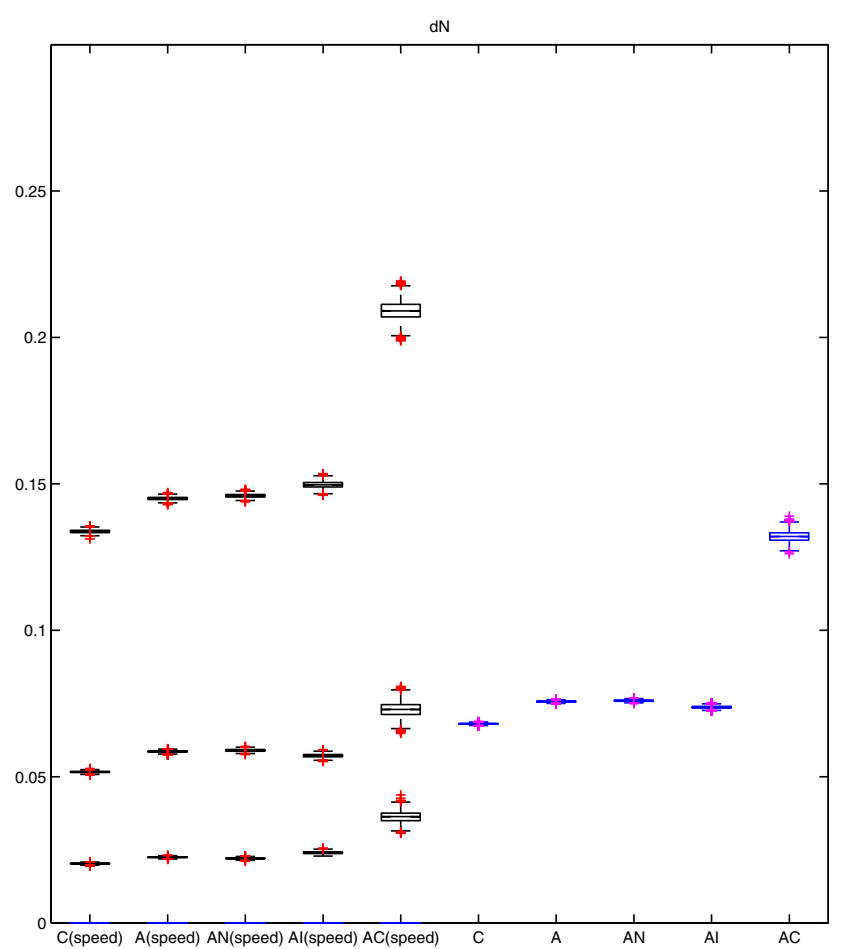

Figure 3

Nonsynonymous substitution rate $\left(d_{N}\right)$ in alignments of constitutive and alternative coding regions of 3029 alternatively spliced genes genes classified by the overall evolution rate. Notation and layout as in Figure 2.

the one that the distribution of $\left|\omega_{\mathrm{C}}-\omega_{\mathrm{AC}}\right|$ was symmetrical, at the significance level $10^{-2}$. Therefore, the detailed analysis of individual genes confirmed the observations made on concatenated alignments.

\section{Discussion}

Evolutionary patterns in different functional regions are known to be significantly different. Conserved genes are duplicated relatively more often [22], although shortly after duplication the evolutionary rate might increase, as the purifying selection is weaker $[23,24]$, and the selection pattern in the two copies may be different [25]. Duret and Mouchiroud [26] observed lower nonsynonymous divergence in genes expressed in multiple tissues when compared to genes with more limited expression patterns, whereas the synonymous substitution rate was roughly the same. Similarly, Pál, Papp and Hurst [27] demonstrated that highly expressed genes tend to be more conserved then genes expressed at a lower level. Our results are consistent with these observations if one assumes that constitutive regions are expressed in more tissues and at a higher rate that alternative ones. Indeed, the former assumption holds for genes with isoforms having clear tis- 


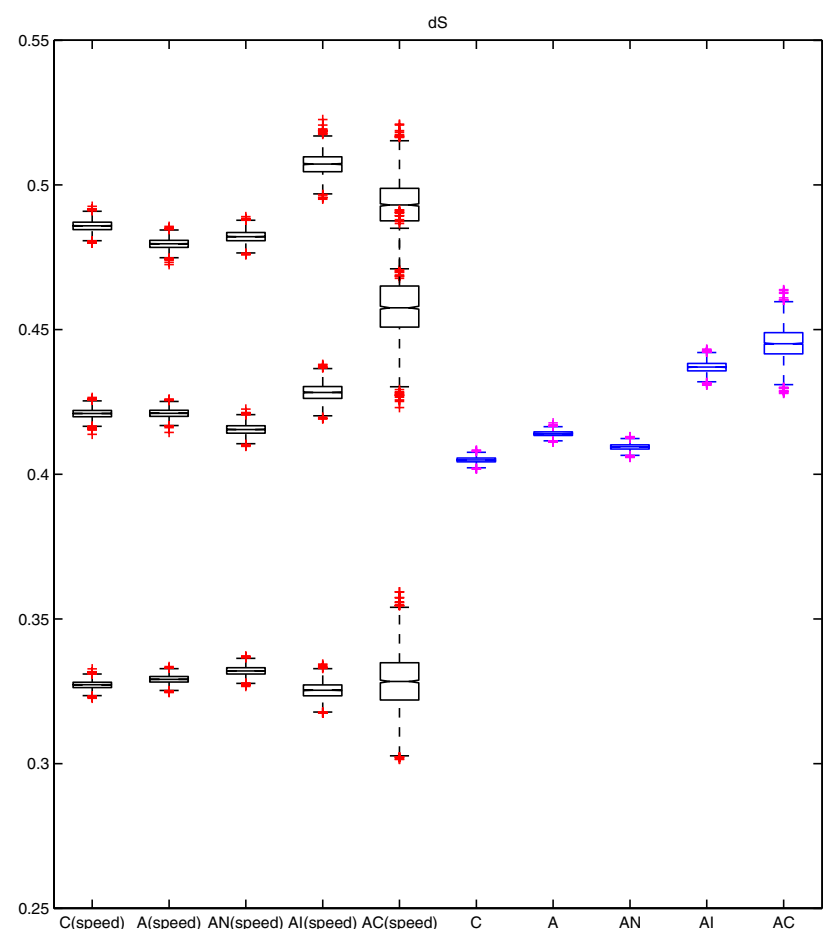

Figure 4

Synonymous substitution rate $\left(d_{s}\right)$ in alignments of constitutive and alternative coding regions of $\mathbf{3 0 2 9}$ alternatively spliced genes classified by the overall evolution rate. Notation and layout as in Figure 2.

sue specific expression pattern, whereas the latter holds for genes with all isoforms expressed ubiquitously.

Young gene regions tend to evolve faster. Several studies [23-25,28] demonstrated post-duplicational relaxation of purifying selection in paralogs. Our results provided evidence of stronger positive selection and/or weaker purifying selection in alternative gene regions.

One possible explanation for our observations could be that the data are contaminated by non-functional isoforms (hence relaxation of purifying selection takes place). We do not believe that to be the case for the following reasons. Firstly, these regions were conserved between human and mouse at a sufficiently high similarity level of $70 \%$ nucleotide identity. Secondly, the observed pattern of increased $\mathrm{d}_{\mathrm{N}}$ level in alternative regions was the most pronounced in 3' (C-terminal) regions, that are the most reliable as regards gene recognition and have higher EST coverage due to polyA-primed ESTs.

As we considered only alternatives derived from RefSeq proteins, we could miss some alternatives and thus label a

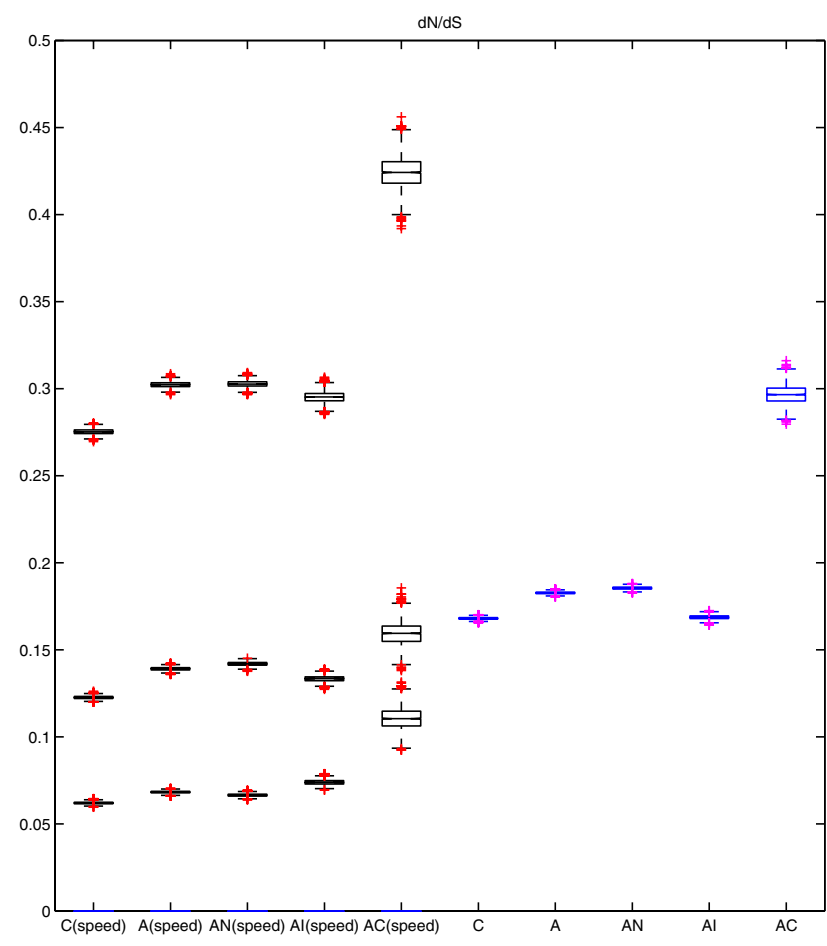

Figure 5

Selection measure $\omega=d_{N} / d_{S}$ in alignments of constitutive and alternative coding regions of 3029 alternatively spliced genes classified by the overall evolution rate. Notation and layout as in Figure 2.

fraction of the alternative regions as constitutive. However, that could only contaminate the constitutive sample with alternative regions and thus blur the observed differences, but not create any spurious effect.

Recently Xing and Lee [21] observed similar rate of nonsynonymous substitutions in alternative and constitutive regions whereas the rate of synonymous substitutions was lower in alternative regions, especially in tissue-specific exons [29]. One possible explanation for that was based on the assumption that conserved alternative exons contain more candidate splicing enhancer sites than constitutive ones [15]. As such sites could be expected to be conserved, like in BRCA1 [19,20], that could lead to higher conservation of synonymous codon positions in alternative regions compared to constitutive ones. However, this explanation seems to be incorrect, since, although indeed $\mathrm{d}_{\mathrm{S}}$ is lower in splicing enhancers, the fractions of constitutive and alternative regions covered by splicing enhancers are the same [30], and if the RNA selection pressure is the same in alternative and constitutive regions, it cannot distort the measurement of $\omega$ [31]. 
Table I: Substitution rates in constitutive and alternative regions of human-mouse concatenated alignments

\begin{tabular}{|c|c|c|c|c|c|}
\hline Concatenate type & $\begin{array}{r}\text { total alignment length, } \\
\text { bp }\end{array}$ & amino-acid identity & $d_{N}$ & $d_{s}$ & $\omega$ \\
\hline \multicolumn{6}{|l|}{ All genes } \\
\hline C & 2822439 & 0.891 & 0.068 & 0.409 & 0.166 \\
\hline A & 3081642 & 0.879 & 0.077 & 0.410 & 0.187 \\
\hline AN & 2194521 & 0.880 & 0.075 & 0.404 & 0.186 \\
\hline $\mathrm{Al}$ & 790026 & 0.884 & 0.074 & 0.421 & 0.176 \\
\hline$A C$ & 97095 & 0.813 & 0.133 & 0.446 & 0.297 \\
\hline \multicolumn{6}{|l|}{ Slow-evolving genes } \\
\hline C & $89747 \mid$ & 0.964 & 0.020 & 0.327 & 0.061 \\
\hline A & 920970 & 0.960 & 0.023 & 0.324 & 0.071 \\
\hline AN & 670623 & 0.961 & 0.022 & 0.323 & 0.068 \\
\hline $\mathrm{Al}$ & 230754 & 0.957 & 0.024 & 0.327 & 0.075 \\
\hline$A C$ & 19593 & 0.940 & 0.036 & 0.329 & 0.111 \\
\hline \multicolumn{6}{|l|}{$\begin{array}{l}\text { Medium-speed } \\
\text { evolving genes }\end{array}$} \\
\hline C & 978984 & 0.913 & 0.052 & 0.422 & 0.123 \\
\hline A & 1092459 & 0.903 & 0.059 & 0.420 & 0.139 \\
\hline AN & 751620 & 0.903 & 0.059 & 0.413 & 0.142 \\
\hline $\mathrm{Al}$ & 313896 & 0.905 & 0.057 & 0.434 & 0.131 \\
\hline$A C$ & 26943 & 0.887 & 0.073 & 0.455 & 0.160 \\
\hline \multicolumn{6}{|l|}{ Fast-evolving genes } \\
\hline C & 945984 & 0.800 & 0.135 & 0.480 & 0.281 \\
\hline A & 1068213 & 0.785 & 0.147 & $0.48 \mathrm{I}$ & 0.306 \\
\hline AN & 772278 & 0.788 & 0.143 & 0.474 & 0.302 \\
\hline $\mathrm{Al}$ & 245376 & 0.787 & 0.148 & 0.504 & 0.294 \\
\hline$A C$ & 50559 & 0.724 & 0.209 & 0.493 & 0.425 \\
\hline
\end{tabular}

C - constitutive regions

A - alternative regions

$\mathrm{AN}-\mathrm{N}$-terminal alternative regions

$\mathrm{Al}$ - internal alternative regions

AC - C-terminal alternative regions

$\mathrm{R}$ - the transition to transvertion rate ratio

$\mathrm{d}_{\mathrm{N}}$ - the nonsynonymous substitution rate

$d_{S}-$ the synonymous substitution rate

$\omega=d_{N} / d_{S}$

However, this effect has not been observed in our study, and the substitution rates differ from those in [21]. As our results are consistent and statistically significant for all classes of genes (fast, medium and slow evolving) and all gene regions ( $\mathrm{N}$-terminal, internal, $\mathrm{C}$-terminal), and do not seem to be caused by contamination, there should be other reasons for this discrepancy. One of them could be the fact that we considered all types of alternatives, as opposed to only cassette exons in other studies. We also considered short alternative regions, its skipping or inclusion might be regulated "outside". Another one could be the use of different methods to calculate the rates of evolution. We used our own implementation of the first method of Ina [32] here, as we needed a tool for very long alignments $\left(\sim 3 \cdot 10^{6} \mathrm{bp}\right)$, whereas Xing and Lee $[21,29]$ used a maximum likelihood method implemented in the PAML package [33]. On the other hand, we considered only RefSeq isoforms and did not distinguish between the minor and major isoform alternatives.
An explanation for our finding could be that the total length of regulatory sites experiencing purifying selection is still small compared to the total length of alternative regions. The pattern of substitutions in insects is less consistent [34]: in N-terminal alternatives, the synonymous rate is higher than in constant regions, whereas in internal alternatives, there are more amino acid substitutions, similar to our observations here.

\section{Conclusion}

Overall, this study corroborates the idea that alternative splicing serves as a testing ground for molecular evolution. Several lines of evidence confirm this hypothesis: (i) alternatively spliced isoforms are often evolutionary young both in mammals [2,10] and in insects [35]; (ii) the rate of nonsynonymous substitutions is higher in alternative regions compared to constitutive ones (this study), (iii) constitutive exons in genes with genome-specific alternative splicing evolve faster than constitutive 


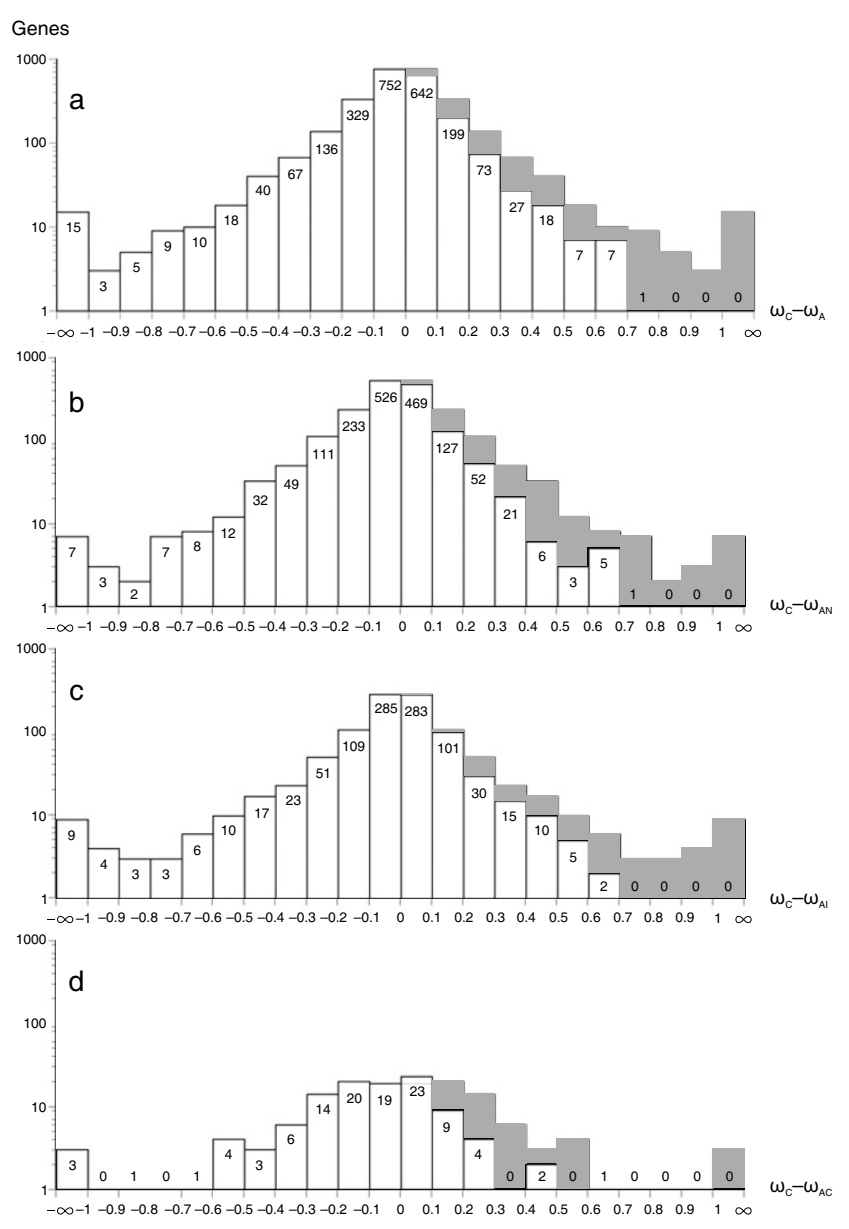

Figure 6

Distributions of $\omega_{\mathbf{C}^{-} \omega_{\mathbf{A}}}, \omega_{\mathbf{C}}-\omega_{\mathbf{A N}}, \omega_{\mathbf{C}}{ }^{-\omega_{\mathbf{A l}}}, \omega_{\mathbf{C}} \mathbf{C}^{-\omega_{\mathbf{A C}}} \cdot \mathbf{a}$. The distribution of $\omega_{C}-\omega_{A}$ for 2358 genes with the total length of human-mouse alignments of constitutive regions and of alternative regions both exceeding 80 bp shows that $\omega$ tends to be larger in alternative regions. In particular, there are 23 genes with $\omega_{C^{-}} \omega_{A}<-0.8$ and no genes with $\omega_{C^{-}} \omega_{A}>0.8$. b, $\mathbf{c}$, d. The distributions of $\omega_{C^{-}} \omega_{A N}, \omega_{C^{-}} \omega_{A}, \omega_{C^{-}} \omega_{A C}$ for genes with long $\mathrm{N}$-terminal ( 1674 genes, top, $\left.\omega_{\mathrm{C}^{-}} \omega_{\mathrm{AN}}\right)$, internal ( 976 genes, middle, $\left.\omega_{C}-\omega_{A 1}\right)$, and $C$-terminal ( 110 genes, bottom, $\omega_{C}-\omega_{A C}$ ) alternative regions, respectively, show that $\omega_{A^{*}}$ tends to be larger than $\omega_{c}$ in all types of alternative regions. the grey shadows are symmetrical to the left parts of the histograms.

regions in genes with conserved structure [36] (cf. a similar observation for duplicated genes [23-25]), (iv) many young (rodent-specific, missing in human and pig as an outgroup) exons are alternatively spliced and tend to have $\omega>1$ in the mouse-rat comparison [30], and (v) the frequency of nonsynonymous SNPs in human genes is higher in alternative regions than in constitutive regions [37].

\section{Methods \\ Definitions}

In an alternatively spliced gene, constitutive regions are defined as the ones that are always exonic and coding, and alternative regions as the ones that are either coding or spliced out. An exon can be either completely constitutive, or completely alternative, or non-coding, or consist of constitutive, alternative and non-coding regions.

A local meta-alignment is a concatenate of all alignment fragments of a fixed type (for example, coding alternative regions) for one particular gene.

A global meta-alignment is the concatenate of local metaalignments of a fixed type for all genes of a fixed group (for example, for all fast-evolving genes).

\section{Data}

Human and mouse mRNA sequences were taken from the NCBI RefSeq database [38] and orthologs were identified and aligned as described previously [39].

Overall, 12356 pairs of orthologous human and mouse genes were considered. The data flow through the analysis pipeline is shown in Figure 1. Out of 12356 human genes, 5754 genes had more than one protein isoform in the EDAS database of alternatively spliced genes [40]. These proteins were mapped to the human-mouse mRNA alignments using Pro-Frame [41] and the results were parsed using a set of Perl scripts that identified constitutive and alternative coding fragments of the human genes and their reading frames. Alternatives confirmed by protein sequences and read in a single frame were identified in 3079 genes. We further restricted the dataset to 3029 pairs with more than $70 \%$ nucleotide identity between the human and mouse genes, as we doubted that the other ones were reliable.

2358 genes were selected for individual substitution rate analysis. These were the ones with both the total length of the human-mouse alignment length of the alternative regions and that of the constitutive regions exceeding 80 base pairs.

\section{Data classification}

We grouped genes with comparable average substitution rates and formed three bins of equal size: slow, mediumspeed, and fast-evolving genes.

We also considered alternative coding regions corresponding to protein $\mathrm{N}$-terminal, middle, and C-terminal parts separately. 


\section{Estimation of substitution rates}

The transitional to transversional substitution rate ratio $R$, as well as thenumbers of synonymous $\left(d_{S}\right)$ and nonsynonymous $\left(\mathrm{d}_{\mathrm{N}}\right)$ substitutions per site were estimated by the Ina method I [32]. Unlike maximum likelihood methods, it afforded considerable results for very long alignments ( $3 \cdot 10^{6} \mathrm{bp}$ ) and it proved to be fast enough to allow bootstrap resampling. We used our own implementation of this method (a set of Perl scripts).

\section{Bootstrapping}

To evaluate the robustness of the estimates for evolutionary parameters of the global meta-alignments, we used bootstrapping to form 2000 alighments of the same length for reach global meta-alignment and estimated amino-acid identity, $\mathrm{d}_{\mathrm{N}^{\prime}} \mathrm{d}_{\mathrm{S}^{\prime}}$ and $\omega=\mathrm{d}_{\mathrm{N}} / \mathrm{d}_{\mathrm{S}}$.

\section{Authors' contributions}

$\mathrm{RN}$ provided the EDAS database. EE and RN analysed the data. MG designed the project. EE and MG wrote the paper. All authors read and approved the final manuscript.

\section{Acknowledgements}

We are grateful to I. King Jordan for alignments of human and mouse mRNAs and to Georgii Bazykin, Alexey Kondrashov, Dmitry Malko, Andrei Mironov, Dmitri Petrov, and Vasily Ramensky for useful discussions.

This study was partially supported by grants from the Ludwig Institute of Cancer Research (CDRF RBO- I 268), the Howard Hughes Medical Institute (55000309), the Russian Fund of Basic Research (04-04-49440), the Russian Academy of Sciences (program "Molecular and Cellular Biology"), and the Russian Science Support Fund.

\section{References}

I. Kriventseva EV, Koch I, Apweiler R, Vingron M, Bork P, Gelfand MS, Sunyaev S: Increase of functional diversity by alternative splicing. Trends Genet 2003, I 9:124-I 28.

2. Modrek B, Lee C): Alternative splicing in the human, mouse and rat genomes is associated with an increased frequency of exon creation and/or loss. Nat Genet 2003, 34:177-I80.

3. Gelfand MS: Computational analysis of alternative splicing. In Handbook of Computational Molecular Biology, Chapman \& Hall/CRC Computer \& Information Science Series Volume 9. Edited by: Alluru S. New York: Chapman \& Hall/CRC; 2005.

4. Kopelman NM, Lancet D, Yanai I: Alternative splicing and gene duplication are inversely correlated evolutionary mechanisms. Nature Genet 2005, 37:588-589.

5. Su Z, Wang J, Yu J, Huang X, Gu X: Evolution of alternative splicing after gene duplication. Genome Res 2006, 16:182-189.

6. Mazumder B, Seshadri V, Fox PL: Translational control by the 3'UTR: the ends specify the means. Trends Biochem Sci 2003, 28:91-98.

7. Pagani F, Baralle FE: Genomic variants in exons and introns: identifying the splicing spoilers. Nat Rev Genet 2004, 5:389-396.

8. Lynch M, Scofield DG, Hong $X$ : The evolution of transcriptioninitiation sites. Mol Biol Evol 2005, 22: I I37-I I 46.

9. Mironov AA, Fickett JW, Gelfand MS: Frequent alternative splicing of human genes. Genome Res 1999, 9:1288-1293.

10. Nurtdinov RN, Artamonova II, Mironov AA, Gelfand MS: Low conservation of alternative splicing patterns in the human and mouse genomes. Hum Mol Genet 2003, I 2: I313-1320.

II. Clark F, Thanaraj TA: Categorization and characterization of transcript-confirmed constitutively and alternatively spliced introns and exons from human. Hum Mol Genet 200I, I I:45 I-464.

12. Burset M, Seledtsov IA, Solovyev VV: Analysis of canonical and non-canonical splice sites in mammalian genomes. Nucleic Acids Res 2000, 28:4364-4375.

13. Sorek R, Ast G: Intronic sequences flanking alternatively spliced exons are conserved between human and mouse. Genome Res 2003, 13:1631-1637.

14. Sorek R, Shemesh R, Cohen Y, Basechess O, Ast G, Shamir R: A nonEST-based method for exon-skipping prediction. Genome Res 2004, I4:1617-1623.

15. Yeo GW, Van Nostrand E, Holste D, Poggio T, Burge CB: Identification and analysis of alternative splicing events conserved in human and mouse. Proc Natl Acad Sci U S A 2005, I 02:2850-2855.

16. Xing Y, Lee C: Assessing the application of $\mathrm{Ka} / \mathrm{Ks}$ ratio test to alternatively spliced exons. Bioinformatics 2005, 2 I :370 I-3797.

17. lida $\mathrm{K}, \mathrm{Akashi} \mathrm{H}$ : A test of translational selection at 'silent' sites in the human genome: base composition comparisons in alternatively spliced genes. Gene 2000, 26 I:93-105.

18. Filip LC, Mundy NI: Rapid evolution by positive Darwinian selection in the extracellular domain of the abundant lymphocyte protein CD45 in primates. Mol Biol Evol 2004, 21:|504-|5||.

19. Hurst LD, Pál C: Evidence for purifying selection acting on silent sites in BRCAI. Trends Genet 200I, I 7:62-65.

20. Orban TI, Olah E: Purifying selection on silent sites - a constraint from splicing regulation? Trends Genet 200 I, I 7:252-253.

21. Xing $Y$, Lee C: Evidence of functional selection pressure for alternative splicing events that accelerate evolution of protein subsequences. Proc Natl Acad Sci U S A 2005, |02:|3526-|353|.

22. Davis JC, Petrov DA: Preferential duplication of conserved proteins in eukaryotic genomes. PLoS Biol 2004, 2:318-326.

23. Kondrashov FA, Rogozin IB, Wolf YI, Koonin EV: Selection in the evolution of gene duplications. Genome Biol 2002, 3:RESEARCH0008.

24. Conant GC, Wagner A: Asymmetric sequence divergence of duplicate genes. Genome Res 2003, I3:2052-2058.

25. Zhang $\mathrm{P}, \mathrm{Gu} \mathrm{Z}$, Li WH: Different evolutionary patterns between young duplicate genes in the human genome. Genome Biol 2003, 4:R56.

26. Duret L, Mouchiroud D: Determinants of substitution rates in mammalian genes: expression pattern affects selection intensity but not mutation rate. Mol Biol Evol 2000, I 7:68-74.

27. Pal C, Papp B, Hurst LD: Highly expressed genes in yeast evolve slowly. Genetics 200I, I 58:927-93I.

28. Jordan IK, Wolf YI, Koonin EV: Duplicated genes evolve slower than singletons despite the initial rate increase. BMC Evol Biol 2004, 4:22.

29. Xing Y, Lee CJ: Protein Modularity of Alternatively Spliced Exons Is Associated with Tissue-Specific Regulation of Alternative Splicing. PLoS Genet 2005, I:e34.

30. Parmley JL, Chamary JV, Hurst LD: Evidence for purifying selection against synonymous mutations in mammalian exonic splicing enhancers. Mol Biol Evol 2006, 23:301-309.

31. Xing Y, Lee C: Can RNA selection pressure distort the measurement of Ka/Ks? Gene in press. 2006, Feb 16

32. Ina $Y$ : New methods for estimating the numbers of synonymous and nonsynonymous substitutions. I Mol Evol 1995, 40:190-226.

33. Yang Z: PAML: a program package for phylogenetics analysis by maximum likelyhood. Comput Appl Biosci 1997, I3:555-556.

34. Ermakova EO, Malko DB, Gelfand MS: Evolutionary differences between alternative and constant protein-coding regions of alternatively spliced Drosophila genes. Biofizika in press.

35. Malko DB, Makeev VJ, Mironov AA, Gelfand MS: Evolution of the exon-intron structure and alternative splicing in fruit flies and malarial mosquito genomes. Genome Res 2006, I6(4):505-9.

36. Cusack BP, Wolfe $\mathrm{KH}$ : Changes in alternative splicing of human and mouse genes are accompanied by faster evolution of constitutive exons. Mol Biol Evol 2005, 22:2198-2208.

37. Ramensky V, Neverov AD, Nurtdinov RN, Mironov AA, Gelfand MS: Human SNPs and alternative splicing. Proceedings of the Second International Moscow Conference on Computational Molecular Biology: I8-2I July 2005; Moscow 2005:3 I7-318. 
38. NCBI Reference Sequence (RefSeq) [http:// www.ncbi.nlm.nih.gov/RefSeq/]

39. Jordan IK, Kondrashov FA, Rogozin IB, Tatusov RL, Wolf YI, Koonin $\mathrm{EV}$ : Constant relative rate of protein evolution and detection of functional diversification among bacterial, archaeal and eukaryotic proteins. Genome Biol 200I, 2:research0053. I-0053.9.

40. EDAS: EST-Derived Alternative Splicing Database [http:// www.belozersky.msu.ru/edas/]

41. Mironov AA, Novichkov PS, Gelfand MS: Pro-Frame: similaritybased gene recognition in eukaryotic DNA sequences with errors. Bioinformatics 200I, 17:13-15.

Publish with Bio Med Central and every scientist can read your work free of charge

"BioMed Central will be the most significant development for disseminating the results of biomedical research in our lifetime. "

Sir Paul Nurse, Cancer Research UK

Your research papers will be:

- available free of charge to the entire biomedical community

- peer reviewed and published immediately upon acceptance

- cited in PubMed and archived on PubMed Central

- yours - you keep the copyright

Submit your manuscript here:

http://www.biomedcentral.com/info/publishing_adv.asp 